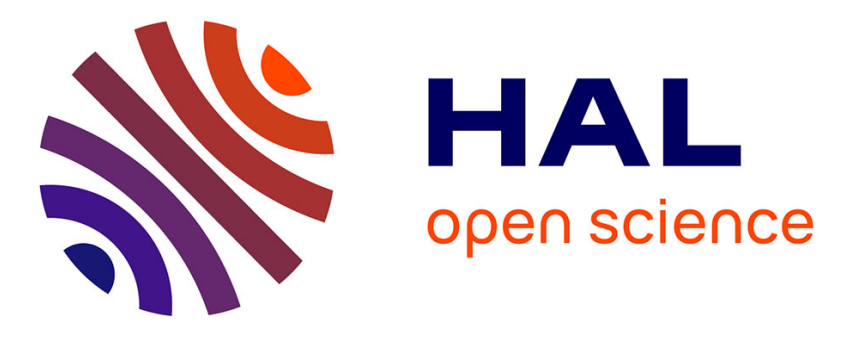

\title{
In vivo pink-beam imaging and fast alignment procedure for rat brain tumor radiation therapy
}

Christian Nemoz, Astrid Kibleur, Jean-Noël Hyacinthe, Gilles Berruyer, Thierry Brochard, Elke Bräuer-Krisch, Géraldine Le Duc, Emmanuel Brun, Hélène Elleaume, Raphä̈l Serduc

\section{To cite this version:}

Christian Nemoz, Astrid Kibleur, Jean-Noël Hyacinthe, Gilles Berruyer, Thierry Brochard, et al.. In vivo pink-beam imaging and fast alignment procedure for rat brain tumor radiation therapy. Journal of Synchrotron Radiation, 2016, 23, pp.339-343. 10.1107/S1600577515018561 . hal-01287950

\section{HAL Id: hal-01287950 https://hal.science/hal-01287950}

Submitted on 14 Mar 2016

HAL is a multi-disciplinary open access archive for the deposit and dissemination of scientific research documents, whether they are published or not. The documents may come from teaching and research institutions in France or abroad, or from public or private research centers.
L'archive ouverte pluridisciplinaire HAL, est destinée au dépôt et à la diffusion de documents scientifiques de niveau recherche, publiés ou non, émanant des établissements d'enseignement et de recherche français ou étrangers, des laboratoires publics ou privés. 


\title{
In vivo pink-beam imaging and fast alignment procedure for rat brain tumor radiation therapy
}

\author{
Christian Nemoz, Astrid Kibleur, Jean Noël Hyacinthe, Gilles Berruyer, \\ Thierry Brochard, Elke Bräuer-Krisch, Géraldine Le Duc, Emmanuel Brun, \\ Hélène Elleaume and Raphaël Serduc
}

J. Synchrotron Rad. (2016). 23, 339-343

\section{IUCr Journals CRYSTALLOGRAPHY JOURNALS ONLINE}

Copyright (C) International Union of Crystallography

Author(s) of this paper may load this reprint on their own web site or institutional repository provided that this cover page is retained. Republication of this article or its storage in electronic databases other than as specified above is not permitted without prior permission in writing from the IUCr.

For further information see http://journals.iucr.org/services/authorrights.html 
JOURNAL OF SYNCHROTRON RADIATION

ISSN 1600-5775

Received 29 July 2015

Accepted 4 October 2015

Edited by M. Yamamoto, RIKEN SPring-8 Center, Japan

Keywords: synchrotron microbeam radiation therapy; pink-beam imaging; alignment procedure; brain tumors.

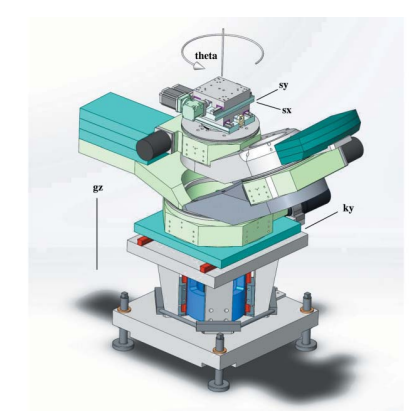

C 2016 International Union of Crystallography

\section{In vivo pink-beam imaging and fast alignment procedure for rat brain tumor radiation therapy}

\author{
Christian Nemoz, ${ }^{a}$ Astrid Kibleur, ${ }^{\text {b,c }}$ Jean Noël Hyacinthe, ${ }^{\text {d,e }}$ Gilles Berruyer, ${ }^{a}$ \\ Thierry Brochard, ${ }^{a}$ Elke Bräuer-Krisch, ${ }^{a}$ Géraldine Le Duc, ${ }^{a}$ Emmanuel Brun, ${ }^{f}$ \\ Hélène Elleaume $^{b, c *}$ and Raphaël Serduc ${ }^{b, c}$
}

\begin{abstract}
${ }^{\mathbf{a}}$ European Synchrotron Radiation Facility, 38043 Grenoble, France, 'bINSERM, U836, F-38043 Grenoble, France, ${ }^{\mathbf{c}}$ Grenoble Institut des Neurosciences, Université Joseph Fourier, UMR-S836, F-38043 Grenoble, France, ${ }^{\mathbf{d}}$ School of Health Sciences - Geneva, University of Applied Sciences and Arts Western, Switzerland, Image Guided Interventions Laboratory, Faculty of Medicine, University of Geneva, Geneva, Switzerland, and 'Department of Physics, Ludwig-Maximilians-University, Garching 85748, Germany. *Correspondence e-mail: h.elleaume@esrf.fr
\end{abstract}

A fast positioning method for brain tumor microbeam irradiations for preclinical studies at third-generation X-ray sources is described. The threedimensional alignment of the animals relative to the $\mathrm{X}$-ray beam was based on the X-ray tomography multi-slices after iodine infusion. This method used pinkbeam imaging produced by the ID17 wiggler. A graphical user interface has been developed in order to define the irradiation parameters: field width, height, number of angles and X-ray dose. This study is the first reporting an image guided method for soft tissue synchrotron radiotherapy. It allowed microbeam radiation therapy irradiation fields to be reduced by a factor of $\sim 20$ compared with previous studies. It permitted more targeted, more efficient brain tumor microbeam treatments and reduces normal brain toxicity of the radiation treatment.

\section{Introduction}

Synchrotron microbeam radiation therapy (MRT) is a method of radiosurgery which is under development at the ID17 biomedical beamline of the European Synchrotron Radiation Facility in Grenoble (Bräuer-Krisch et al., 2010). Preclinical results over 15 years led our teams to prepare a clinical transfer of this technology and the foreseen medical applications will be focused on neuroscience diseases (Grotzer et al., 2015).

MRT is based on very high radiation doses (kGy) delivered through a lattice of parallel $50 \mu \mathrm{m}$-wide microbeams spaced $200 \mu \mathrm{m}$ apart. This particular radiation geometry and the unusual radiation dose require very precise targeting while sparing normal tissue neighboring the lesion (Laissue et al., 1998; Serduc et al., 2006). We recently developed a fast and efficient image-guided method for preclinical epilepsy treatment in rats based on radio-projection of the skull (Serduc et al., 2010a). Beam positioning was defined according to stereotactic coordinates from the bregma, easily distinguished on pink-beam (heavily filtered low-energy white beam) absorption images. GAERS rats (Marescaux et al., 1992) were successfully treated using this alignment method (Pouyatos $e t$ al., 2013). However, this method is not applicable to brain tumor MRT because no contrast can be obtained from the tumor using absorption projections. In this paper, we describe how we derived our pink-beam alignment method (Serduc et $a l ., 2010 a$ ) into a brain tumor conformal irradiation tool based on micro-tomographic images. 
The reduction of the radiation fields in preclinical MRT appears mandatory to demonstrate the relevance of MRT for brain tumors treatment. Therapeutic efficacy might be hidden by side-effects induced on irradiated normal brain. Until now, MRT protocols usually covered at least half of the animal brain volume (Serduc et al., 2009; Bouchet et al., 2013). For instance, the ratio of the tumor volume to the cross-fired beam volume has been estimated to be around 0.06 by Laissue et al. (1998). In order to reduce brain toxicity and increase future MRT efficiency, an image-guided protocol was developed based on iodine-loaded brain tumors Computed Tomography (CT). Iodine is known to accumulate in brain tumor parenchyma when intravenously injected and it is an efficient and routinely used X-ray contrast agent in clinic (Adam et al., 2003). Furthermore, the irradiation of iodine accumulated in the tumor will generate a local dose enhancement in the lesion via photo-activation (Adam et al., 2006). Using this new protocol, irradiation fields were adapted to the lesion size and margins for each irradiation port on tomographic images and 9L tumors implanted in rat brains were treated by MRT. We also evaluated phase extraction computed tomography in order to improve tumor visualization and targeting. The effects of irradiation volume on rat survival were evaluated after large fields MRT and two different configurations of conformal MRT which relies on our alignment method. The term 'conformal' refers to irradiations where the irradiation field is adapted to the tumor size. The interspersed MRT method is described by Bräuer-Krisch et al. (2013) and is based on two, orthogonally crossfired, arrays of parallel non-intersecting and mutually interspersed microbeams.

\section{Material and methods}

\subsection{Alignment, imaging and irradiation protocols}

All the animal care procedures conformed to the Guidelines of the French Government (licenses 380324 and A3818510002). Experiments were performed under inhalation anesthesia, $5 \%$ isoflurane for induction and $2.5 \%$ isoflurane for CT imaging and MRT treatment.

\subsection{Tumor model}

Gliosacorma cells (9LGS) (Benda et al., 1971) were injected following the protocol described by Serduc et al. (2009). In short, $10^{4}$ cells in suspension in $1 \mu \mathrm{L}$ of cell culture medium were injected into the right striatum of 26 Fischer rat brains (10 weeks old). The coordinates of injection were defined according to the Paxinos Atlas $[3.5 \mathrm{~mm}$ laterally from the bregma and $5.5 \mathrm{~mm}$ deep (Paxinos \& Watson, 2004)].

\subsection{Radiation source and alignment system}

The X-ray source was the ID17 biomedical beamline of the ESRF in Grenoble, France. A 21-pole wiggler is installed on the electron beam at the ID17 port. The vertical gap between the two opposite multipole rows can be remotely adjusted, thus permitting the photons flux and the energy spectrum to vary drastically. A variety of attenuators $(\mathrm{C}, \mathrm{Al}, \mathrm{Cu}, \mathrm{Au})$ with different thicknesses can also be inserted into the X-ray beam to reduce the flux and/or adjust the energy spectrum. Last, several slit systems, remotely adjustable, permit the horizontal and vertical sizes of the beam to be precisely defined to match the tumor shape. The multislit collimator used to produce the microbeams can also be inserted or removed from the beam by remote control. In addition, a two-dimensional X-ray detector setup is installed about $2 \mathrm{~m}$ downstream of the animal goniometer support. The detector is a Fast Readout Low Noise camera (FReLoN; Coan et al., 2006) based on a $2048 \times$ 2048 CCD chip coupled with a scintillator and an optical lenses system. The detector pixel size can be electronically reduced (binning) to obtain a good compromise between a sub-millimetric resolution $($ e.g. $100 \mu \mathrm{m})$ and a reasonable amount of data. The field of view can be up to $50 \mathrm{~mm}$. This setup can be automatically translated into/out of the beam path. The main idea to achieve sub-millimetric precision alignment of the animal in the irradiation beam is to use the same beam for performing both irradiation and X-ray imaging. The beam characteristics should be changed in a very short time ( $<2 \mathrm{~min})$ to switch from typical irradiation mode to conventional radiographic dose imaging mode. In irradiation mode, the minimum wiggler gap $(24.8 \mathrm{~mm})$ is set to benefit from the maximal photon flux, and the attenuators are set to obtain the standard MRT spectrum [typically 50-350 keV with a median energy at $83 \mathrm{keV}$ (Fig. 1)]. The slits are adjusted to the tumor size, the multislit collimator is inserted into the beam, and the FReLoN camera is removed toward a radiation-protecting lead cabinet. In imaging mode, the slits are fully open to profit from the maximum field of view, typically $50 \mathrm{~mm} \times 2 \mathrm{~mm}$, the multislit collimator is removed, the FReLoN detector is inserted, the wiggler gap and the attenuators' thicknesses are adjusted using an in-house code that gives the corresponding beam spectra and flux to obtain a quasi-monochromatic - so-called 'pink beam' - X-ray imaging beam. Assuming that the geometric properties of the beam do not change during these transformations, it is then possible to visualize, measure and adjust the path of the irradiation beam within the animal. The contrast agent used in the experiment was iodine, whose $K$-edge is at $33.17 \mathrm{keV}$. Therefore, the pink-

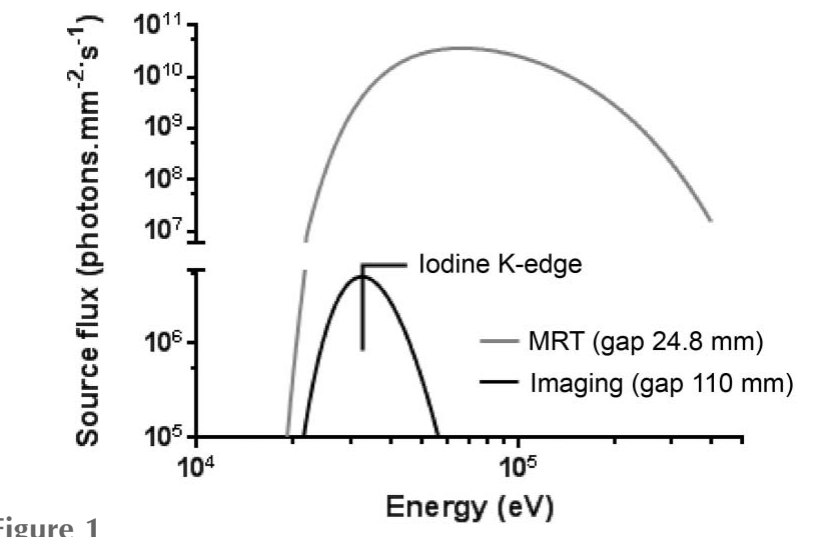

Spectrum and corresponding attenuation range used for imaging and irradiation. Pink-beam imaging (grey) and microbeam radiation (black) spectra used on ID17 for brain tumors alignment and treatment. 
beam average energy range should be tuned above $33.17 \mathrm{keV}$ to optimize the contrast between the iodine-loaded tumors and surrounding tissues. The X-ray dose delivered during the imaging procedure was comparable with a standard X-ray CT scanner dose. These two conditions are reached by choosing $1.5 \mathrm{~mm}$ of $\mathrm{Cu}$ and $1.52 \mathrm{~mm}$ of $\mathrm{Al}$ as beam filtration and the wiggler gap at $110 \mathrm{~mm}$ which gives a spectrum ranging from 22 to $56 \mathrm{keV}$ (centered on the iodine $K$-edge).

\subsection{Imaging and alignment method}

To achieve tumor conformal irradiation, it was necessary to extend the pink-beam imaging method previously developed (Serduc et al., 2010a) from planar radiographic imaging to three-dimensional CT imaging. The contrast agent was delivered by injecting $2 \mathrm{~mL}$ of Iomeron ${ }^{\circledR}$ (400 mg of iodine $\mathrm{ml}^{-1}$ ) via the tail vein. Then the animal was placed on a purposebuilt frame in the ID17 hutch, fixed on a three-axis Kappatype goniometer (Huber, Germany; Fig. 2). This system allows the rotation of the sample in the beam around a vertical axis up to $180^{\circ}$ (theta). An auxiliary motor $\mathbf{k y}$ permits the rotation axis to translate laterally for alignment purpose. In addition, the sample can be centered within the rotation plan via two orthogonal axes (sx, sy), and finally the sample can be translated vertically by means of the same motor used to perform the irradiation (gz).

In order to reduce the acquisition time and the data flow, a region of interest $(\mathrm{ROI})$ is defined on the detector to record only the part of the detector containing the $50 \mathrm{~mm} \times 2 \mathrm{~mm}$ beam, i.e. $512 \times 20$ pixels. To produce the three-dimensional

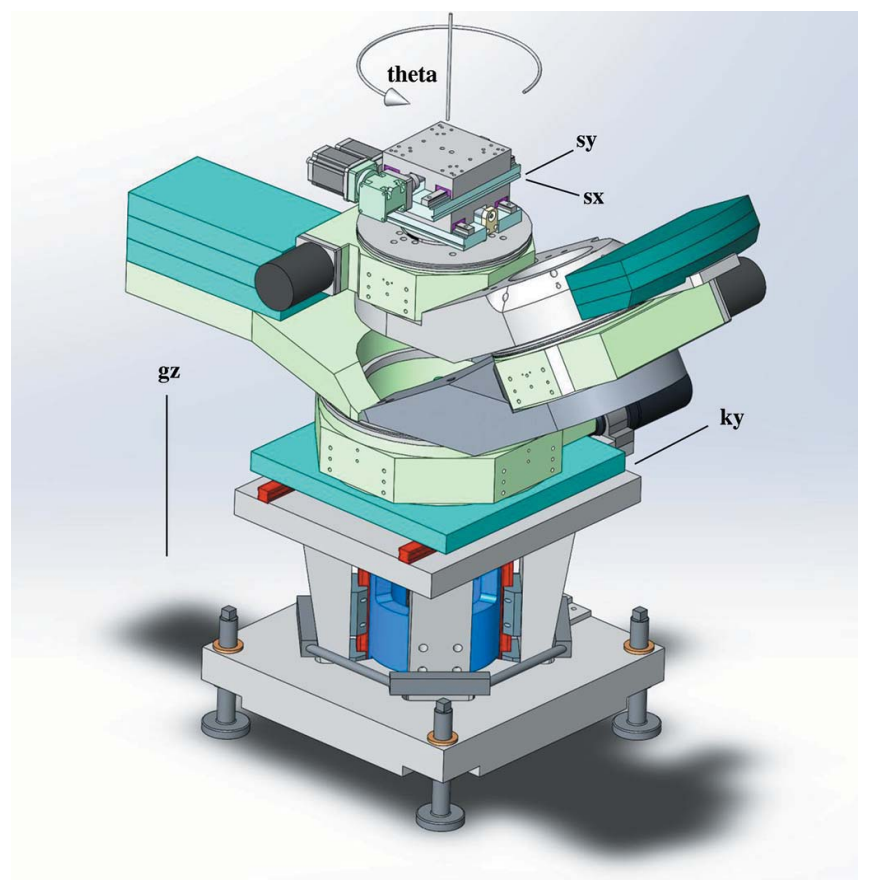

Figure 2

Kappa goniometer. The support at the top is fixed on a two-axis translation system $(\mathbf{s x}, \mathbf{s y})$ to position the sample relative to the tomography rotation axis (theta). In addition, the rotation axis can be moved relative to the beam (ky), and finally the sample can be positioned in the vertical with the $\mathbf{g z}$ vertical translation. volume, the tomography data were recorded at each vertical $2 \mathrm{~mm}$ gz stage by rotating the sample at a typical theta speed of $6^{\circ} \mathrm{s}^{-1}$. The data were acquired on the FReLoN in synchronization with the theta position. The sinogram for one stage consists of a 512 pixels $\times 1000$ angular projections. At each projection, the ROI containing the 20 horizontal slices is recorded. To obtain a suitable vertical range within the animal head (e.g. $>10 \mathrm{~mm}$ ), the CT rotation was performed at five $\mathbf{g z}$ stage positions. Including the time for motors to move and data to be recorded, the total three-dimensional scan lasts around $8 \mathrm{~min}$.

\subsection{Tomography analysis and irradiation}

The sinograms were calibrated with the incident beam previously recorded and the images slices were reconstructed online by dedicated in-house software, called HST (High Speed Tomography) (Mirone et al., 2014). The $\sim 100$ slices (10 $\mathrm{mm}, 100 \mu \mathrm{m}$ step) were then piled up and the resulting volume images sent to the control system. An in-housedeveloped graphical user interface was used to position manually the target volume. Several graphical tools were developed to define the targeted region: as shown in Fig. 3, it is possible to select the vertical start and stop gz (1) of the irradiation, the angle of incidence theta (2), the centering positions sx, sy (3) and the width of the incident beam (4). After validation, the goniometer motors are moved to the position chosen for the first irradiation port. The whole system is then set in irradiation mode by moving simultaneously all the motors to their respective positions: wiggler gap to $24.8 \mathrm{~mm}$, FReLoN detector out, multislit in, horizontal slits to the irradiation position (first port), gz to the vertical start position. The irradiation is ready to be performed. For each conformal irradiation port, theta, sx, sy, and the horizontal slits are moved to their respective positions as computed within the graphical image display interface.

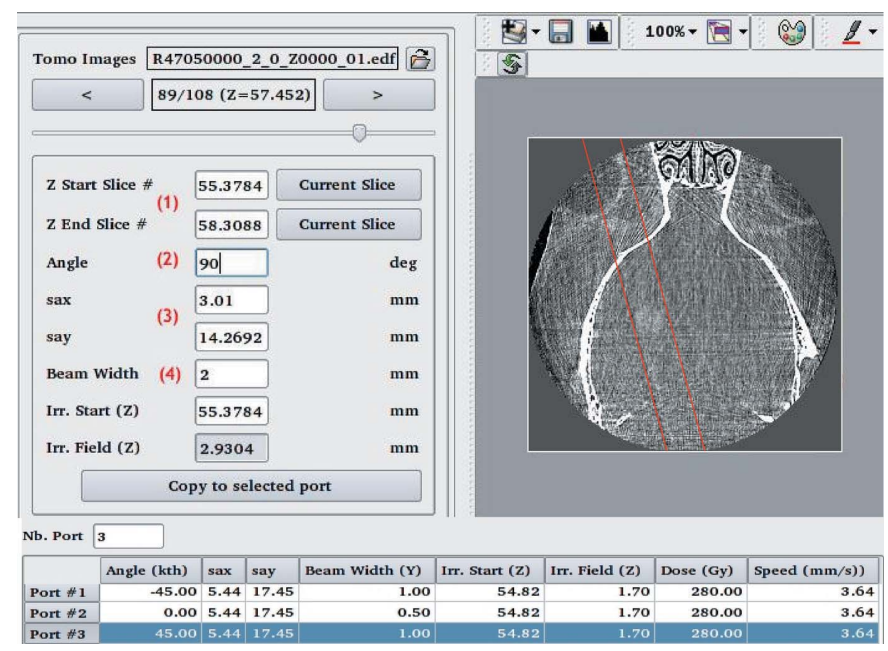

Figure 3

Details of the graphical user interface: visualization of the tomographic slice permits tuning of the irradiation field and orientation of the beam, symbolized here by the red lines. (1): vertical field. (2): incidence. (3): position relative to rotation axis. (4): beam width (horizontal field). The results are transferred to the irradiation sequencer displayed below. 
3. Phase extraction of pink-beam CT: towards higher contrast images for better diagnosis of brain tumors

In order to further evaluate the image quality of the pinkbeam tomographic alignment procedure, a post-processing phase-retrieval algorithm was applied (Paganin et al., 2002) on the acquired images. The tomographic set-up configuration mode used in this study is indeed a propagation-based imaging (sometime referred as in-line tomography) (Cloetens et al., 1996) with a sample-to-detector distance of $4.5 \mathrm{~m}$. In this configuration the quasi-coherent radiation illuminates the object producing a spatially variant phase shift of the X-ray beam. As the X-rays are passing through the sample, parts of the wavefront that have been deflected generate characteristic patterns enlightening the contour of the objects. Due to Fresnel diffraction, the phase shifts are transformed into measurable intensity variations, which are recorded by a detector. The algorithm (Paganin et al., 2002) is a low-pass filter that takes as information the complex refractive index distribution within the sample. The results of the comparison between the CT reconstructed slices of the signal recorded from the detector (referred to as the raw signal) and phase extracted slices are shown in Fig. 4. In these images, the bone has been segmented and a soft tissue windowing is applied. It is obvious that the phase-retrieved image is of superior quality and significant differences in signal-to-noise tatio and contrast enhancement were obtained ( $\mathrm{t}$-test, $p<0.001)$. These results are of high interest because the tumors are more visible and (a)

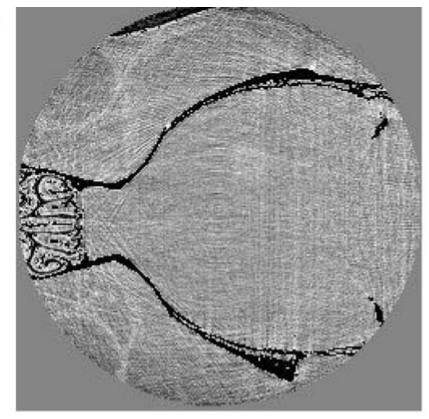

(c)

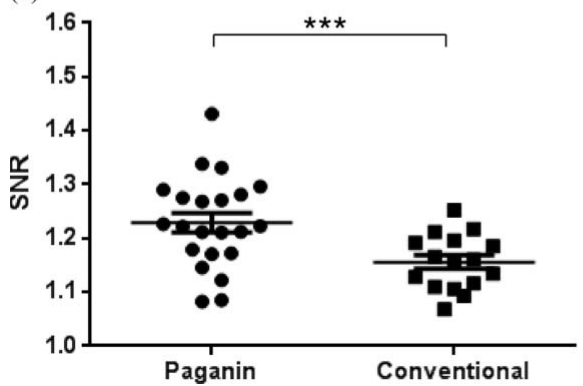

(b)

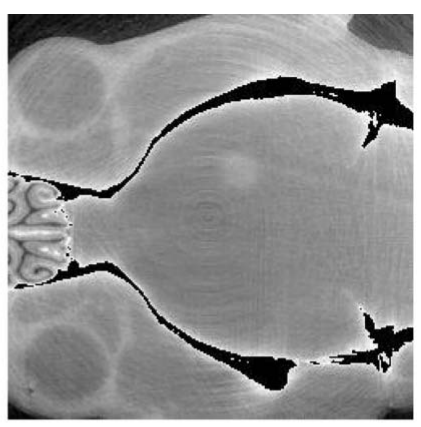

phase-retrieval pink-beam tomography will be used on ID17 for image-guided MRT, for both preclinical MRT studies and future phase I clinical trials.

\section{MRT brain tumor conformal irradiations}

As stated in the Introduction, this fast new method of contrast-agent-loaded tumor tomography opens new possibilities in tumor treatment by synchrotron X-rays. Indeed, the first aim of this method development was to reduce MRT irradiation volumes in preclinical brain tumor treatments. Previous MRT studies showed that normal tissue surrounding the lesion exposed to crossfired irradiations are damaged by the irradiation (Laissue et al., 1998). On the reconstructed CT slices obtained in our study, the measured average tumor volume at imaging time was $4.80 \pm 4.00 \mathrm{~mm}^{3}$ (ranging from $0.97 \mathrm{~mm}^{3}$ to $18.49 \mathrm{~mm}^{3}$ ). By treating brain tumors using the standard $10 \mathrm{~mm} \times 10 \mathrm{~mm}$ field, a $1000 \pm 0 \mathrm{~mm}^{3}$ irradiation volume would have been generated in crossfired regions but, actually, during one irradiation, the $z$-motor accidentally stopped at $9.15 \mathrm{~mm}$ instead of $10 \mathrm{~mm}$ yielding a mean irradiation field of $877 \pm 69 \mathrm{~mm}^{3}$ for this group. The crossfire irradiated fraction (brain region where the beams from different ports intersect) was reduced by a factor of 16-20 when conformal irradiations were used for tumor irradiation. The tumor volume to irradiation volume ratio dropped from 190 to 11.7 and 9.5 when conformal MRT and conformal interspersed MRT were used.

Then, the influence of the field size was evaluated by irradiating tumorbearing rats according to three different irradiation geometries [Figs. 5(a)-5(c)]. Rat survivals were compared after (i) large-field MRT irradiations mainly used in preclinical studies $(8 \mathrm{~mm} \times$ $10 \mathrm{~mm} \times 10 \mathrm{~mm}$ ), (ii) conformal MRT irradiation where the width and height of the beam were adapted to tumor margins, and (iii) interspersed MRT (Bräuer-Krisch et al., 2013) where irradiation fields were adjusted to tumor size and $50 \mu \mathrm{m}$-thick horizontal microbeams were interspersed by $100 \mu \mathrm{m}$ and $90^{\circ}$.

The survival results of the irradiated 9L-bearing rats are shown in Fig. 5(d). Median survival times were 16.0, 39.0, 88 and 154.0 days after implantation for the control, large-field, conformal and interspersed groups, respectively. The corresponding increases in life span (ILS) were $143 \%, 450 \%$ and $1220 \%$ compared with control groups. To our knowledge, these two latter ILSs are the largest ever reported for 9L tumor MRT treatment. MRT has been shown to be more effective on brain tumor than 


\begin{tabular}{|c|c|c|c|c|}
\hline (d) Tumor size at imaging & \multicolumn{4}{|c|}{$4.80 \pm 4.00 \mathrm{~mm}^{3}\left[0.97: 18.49 \mathrm{~mm}^{3}\right]$} \\
\hline Groups & Ctrl & LF MRT (A) & Conf MRT (B) & Int. MRT (C) \\
\hline $\mathbf{n}$ & 7 & 6 & 6 & 6 \\
\hline Ir fields $\left(\mathrm{mm}^{3}\right)$ & N.A & $877 \pm 69$ & $54 \pm 51$ & $44 \pm 48$ \\
\hline Peak Dose (Gy) & 0 & $332 \pm 20$ & $630 \pm 154$ & $150 \pm 0$ \\
\hline MST (days) & 16 & 39 & 88 & 154 \\
\hline ILS (\%) & 100 & 143 & 450 & 1220 \\
\hline Ir Vol / Tum Vol & N.A & 190 & 11.7 & 9.5 \\
\hline
\end{tabular}

Figure 5

Microbeam radiation therapy irradiation modes. Brain tumor irradiations were performed using empirical irradiation fields [vertical microbeams, $10 \mathrm{~mm} \times 10 \mathrm{~mm} \times 10 \mathrm{~mm}(a)$ ], using conformal MRT [Conf MRT $(b)$ ] where beam height and width were adapted to tumor size, and interspersed MRT [Int MRT $(c)$ ] where horizontal microbeams were interlaced at the tumor site and the irradiation field fitted to the tumor volume. The table $(d)$ shows the averages of irradiation field sizes and their effects on brain tumor bearing rat median survival time (MST) and increase in life span (ILS).

conventional X-rays using no contrast agent (Dilmanian et al., 2002) but its potential has actually been underestimated because of the large irradiation fields used in previous MRT experiments. Indeed, by reducing the irradiation fields to tumor shape and by optimizing irradiation geometries [interlaced (Serduc et al., 2010b), interspersed (Bräuer-Krisch et al., 2005) etc.], we demonstrate in this study that MRT can be much more efficient in brain tumor palliation than previously suggested. The reduction of the normal tissue crossfired regions [neighboring the tumor and radio-injured (Laissue et al., 1998; Bouchet et al., 2010)] decreased sub-acute normal tissue toxicity and contributed to the increase in ILS obtained after conformal MRT compared with the large-field MRT. We strongly recommend using this alignment method and conformal irradiation for future MRT experiments, especially when survival curves are published. Human microbeam irradiations performed in the frame of our future MRT phase I clinical trial will obviously require conformal exposures, and a significant contribution of conformal MRT in tumor control can be reasonably expected when delivered as part of a conventional treatment.

\section{Acknowledgements}

The authors acknowledge the support of the ID17 staff and A. Bravin for providing in-house research beam time at the ESRF Biomedical beamline. E. Brun thanks Pôle-Emploi France for financial support. The authors thank the Conseil Régional Rhône-Alpes, Ligue contre le Cancer, comité de la Drôme, l'Association pour la Recherche contre le Cancer and ANR-11-LABX-0063/ANR-11-IDEX-0007.

\section{References}

Adam, J. F., Elleaume, H., Le Duc, G., Corde, S., Charvet, A. M., Troprès, I., Le Bas, J. F. \& Estève, F. (2003). J. Cereb. Blood Flow Metab. 23, 499-512.

Adam, J. F., Joubert, A., Biston, M. C., Charvet, A. M., Peoc'h, M., Le Bas, J. F., Balosso, J., Estève, F. \& Elleaume, H. (2006). Int. J. Radiat. Oncol. Biol. Phys. 64, 603-611.

Benda, P., Someda, K., Messer, J. \& Sweet, W. H. (1971). J. Neurosurg. 34, 310-323.

Bouchet, A., Lemasson, B., Christen, T., Potez, M., Rome, C., Coquery, N., Le Clec'h, C., Moisan, A., Bräuer-Krisch, E., Leduc, G., Rémy, C., Laissue, J. A., Barbier, E. L., Brun, E. \& Serduc, R. (2013). Radiother. Oncol. 108, 143-148.

Bouchet, A., Lemasson, B., Le Duc, G., Maisin, C., Bräuer-Krisch, E., Siegbahn, A. E., Renaud, L., Khalil, E., Rémy, C., Poillot, C., Bravin, A., Laissue, J. A., Barbier, E. L. \& Serduc, R. (2010). Int. J. Radiat. Oncol. Biol. Phys. 78, 1503-1512.

Bräuer-Krisch, E., Nemoz, C., Brochard, T., Berruyer, G., Renier, M., Pouyatos, B. \& Serduc, C. (2013). J. Phys. Conf. Ser. 425, 022001.

Bräuer-Krisch, E., Requardt, H., Régnard, P., Corde, S., Siegbahn, E., LeDuc, G., Brochard, T., Blattmann, H., Laissue, J. \& Bravin, A. (2005). Phys. Med. Biol. 50, 3103-3111.

Bräuer-Krisch, E., Serduc, R., Siegbahn, E. A., Le Duc, G., Prezado, Y., Bravin, A., Blattmann, H. \& Laissue, J. A. (2010). Mutat. Res. Rev. Mutat. Res. 704, 160-166.

Cloetens, P., Barrett, R., Baruchel, J., Guigay, J. P. \& Schlenker, M. (1996). J. Phys. D, 29, 133-146.

Coan, P., Peterzol, A., Fiedler, S., Ponchut, C., Labiche, J. C. \& Bravin, A. (2006). J. Synchrotron Rad. 13, 260-270.

Dilmanian, F. A., Button, T. M., Le Duc, G., Zhong, N., Pena, L. A., Smith, J. A., Martinez, S. R., Bacarian, T., Tammam, J., Ren, B., Farmer, P. M., Kalef-Ezra, J., Micca, P. L., Nawrocky, M. M., Niederer, J. A., Recksiek, F. P., Fuchs, A. \& Rosen, E. M. (2002). Neuro-Oncol. 4, 26-38.

Grotzer, M. A., Schültke, E., Bräuer-Krisch, E. \& Laissue, J. A. (2015). Phys. Med. 31, 564-567.

Laissue, J. A., Geiser, G., Spanne, P. O., Dilmanian, F. A., Gebbers, J. O., Geiser, M., Wu, X. Y., Makar, M. S., Micca, P. L., Nawrocky, M. M., Joel, D. D. \& Slatkin, D. N. (1998). Int. J. Cancer, 78, 654-660. Marescaux, C., Vergnes, M. \& Depaulis, A. (1992). J. Neural Transm. Suppl. 35, 37-69.

Mirone, A., Brun, E., Gouillart, E., Tafforeau, P. \& Kieffer, J. (2014). Nucl. Instrum. Methods Phys. Res. B, 324, 41-48.

Paganin, D., Mayo, S. C., Gureyev, T. E., Miller, P. R. \& Wilkins, S. W. (2002). J. Microsc. 206, 33-40.

Paxinos, G. \& Watson, C. (2004). The Rat Brain in Stereotaxic Coordinates. New York: Academic Press.

Pouyatos, B., Serduc, R., Chipaux, M., Chabrol, T., Bräuer-Krisch, E., Nemoz, C., Mathieu, H., David, O., Renaud, L., Prezado, Y., Laissue, J. A., Estève, F., Charpier, S. \& Depaulis, A. (2013). Neurobiol. Dis. 51, 152-160.

Serduc, R., Berruyer, G., Brochard, T., Renier, M. \& Nemoz, C. (2010a). J. Synchrotron Rad. 17, 325-331.

Serduc, R., Bräuer-Krisch, E., Bouchet, A., Renaud, L., Brochard, T., Bravin, A., Laissue, J. \& Le Duc, G. (2009). J. Synchrotron Rad. 16, 587-590.

Serduc, R., Bräuer-Krisch, E., Siegbahn, E. A., Bouchet, A., Pouyatos, B., Carron, R., Pannetier, N., Renaud, L., Berruyer, G., Nemoz, C., Brochard, T., Rémy, C., Barbier, E. L., Bravin, A., Le Duc, G., Depaulis, A., Estève, F. \& Laissue, J. A. (2010b). PLoS One, 5, e9028.

Serduc, R., Vérant, P., Vial, J. C., Farion, R., Rocas, L., Rémy, C., Fadlallah, T., Brauer, E., Bravin, A., Laissue, J., Blattmann, H. \& van der Sanden, B. (2006). Int. J. Radiat. Oncol. Biol. Phys. 64, 1519-1527. 